\title{
Geometric shapes and relationships of some one-body and multi-body leptodermous distributions
}

\author{
G. Royer, N. Mokus and J. Jahan \\ Laboratoire Subatech, UMR: IN2P3/CNRS-Université-IMT, Nantes 44, France
}

\begin{abstract}
Different families of geometric shapes, derived mainly from lemniscatoids, are proposed to describe ground and excited states of leptodermous distributions of nuclear matter. The transition from one spherical or ellipsoidal nucleus to several spherical or ellipsoidal nuclei or vice versa (in the decay and entrance channels of nuclear reactions: fission, fusion and fragmentation) is particularly investigated. The geometric characteristics of these configurations are given, allowing the calculations of the system energy, of the dynamics of the reactions and of the angular distribution of the fragments.

PACS numbers: 24.75.+i, 21.60.Gx, 23.60.+e, 02.40.-k, 02.40.Re
\end{abstract}

\section{INTRODUCTION}

In nuclear and particle physics, astrophysics, molecular physics, mechanics and in other various scientific domains it is useful to simulate physical leptodermous (thin skin) distributions of matter or charge by geometric shapes, and, to know their main characteristics such as center of inertia, volume, surface, curvature, moments of inertia, quadrupole moment,... Assuming volume conservation during deformation, the spherical form minimizes the surface energy but maximizes the Coulomb repulsion (as in atomic nuclei) or the gravitational attraction (as in stars and galaxies). The rotation of these physical objects may induce large ellipsoidal deformations (super and hyperdeformations of rotating nuclei, planets, ellipsoidal galaxies,...). Transition from one-body to two-body or several body shapes may also occur by very rapid rotation or decay of excited nuclear systems (binary and ternary fission, fragmentation in heavy-ion reactions at intermediate energies,...). Conversely, separated nuclei or astrophysical objects may also fuse (fusion of two heavy ions, fusion of three $\alpha$ particles submitted to high pressure in stars,...).

The nuclear phenomena of large amplitude have been described within different hypotheses and various models; mainly macroscopic-microscopic Liquid Drop Models [1-5] and cluster models ([5], dinuclear model [6], dynamical cluster-decay model $[7], \ldots)$.

The purpose of the present work is to provide geometric shapes useful to carry on the description of the $\alpha$ and cluster radioactivities [5, 8], fusion [3], fission [4, 9-12], fragmentation processes [13] and normal, super and hyperdeformed states [14]. The main characteristics of these shapes are given in order to determine the energy of the nuclear systems, the dynamics of the reactions and eventually the angular distribution of the fragments. After recalling general definitions, the following shapes will be successively investigated: ellipsoids, symmetric and asymmetric elliptic and hyperbolic lemniscatoids, prolate symmetric and asymmetric compact ternary shapes, toroids and bubbles.

Other planar and three-dimensional multibody shapes such as linear chain, triangle, square, tetrahedron, pentagon, trigonal bipyramid, square pyramid, hexagon, octahedron, octagon and cube have also been used to describe some light nuclei as $\alpha$ molecules [15].

\section{GENERAL DEFINITIONS}

For leptodermous distributions of constant matter or charge density, liquid drop models have been developped and the total energy of the systems is mainly the sum of the volume, surface, curvature and Coulomb energies. The relative (to the sphere of radius $R_{0}$ ) shape-dependent surface $B_{s}$, curvature $B_{k}$ and Coulomb (or gravitational) $B_{C}$ functions are respectively given by $[16-19]$

$$
\begin{aligned}
B_{s} & =\int_{\sigma} \frac{d \sigma}{4 \pi R_{0}{ }^{2}}, \\
B_{k} & =\int_{\sigma} k_{l} \frac{d \sigma}{8 \pi R_{0}},
\end{aligned}
$$

where $k_{l}$ is the mean local curvature of the surface given in terms of the principal curvature radii $R_{1}$ and $R_{2}$ by $k_{l}=R_{1}^{-1}+R_{2}^{-1}$.

$$
B_{C}=\frac{15}{16 \pi^{2} R_{0}{ }^{5}} \int d \tau \int \frac{d \tau^{\prime}}{\left|\vec{r}-\vec{r}^{\prime}\right|}
$$


which for axially symmetric shapes reduces to

$$
B_{C}=\frac{1}{2} \int \frac{v\left(\theta_{i}\right)}{v_{0}}\left[\frac{R\left(\theta_{i}\right)}{R_{0}}\right]^{3} \sin \left(\theta_{i}\right) d \theta_{i} .
$$

The potential $v(\theta)$ is calculated at the surface of the shape and $v_{0}$ is the surface potential of the sphere

$$
\frac{v\left(\theta_{i}\right)}{v_{0}}=\frac{3}{2 \pi R_{0}^{2}} \int \frac{\rho\left[\left(\rho_{i}+\rho\right) \frac{d z}{d \theta}+\left(z_{i}-z\right) \frac{d \rho}{d \theta}\right] K(k)-\frac{1}{2}\left[\left(\rho_{i}+\rho\right)^{2}+\left(z_{i}-z\right)^{2}\right] \frac{d z}{d \theta} D(k)}{\sqrt{\left(\rho_{i}+\rho\right)^{2}+\left(z_{i}-z\right)^{2}}} d \theta,
$$

where $\rho=R(\theta) \sin \theta, z=R(\theta) \cos \theta$ and $D(k)$ and $K(k)$ are deduced from elliptic integrals. These dimensionless shape-dependent $B_{s}, B_{k}$ and $B_{C}$ functions are equal to one for the spherical configuration.

To determine the angular distribution of the fragments in fission, the knowledge of the inverse effective moment of inertia is needed. For axially symmetric shapes it reads

$$
I_{e f f}^{-1}=I_{\|}^{-1}-I_{\perp}^{-1}
$$

where $I_{\|}$and $I_{\perp}$ are respectively the parallel and perpendicular moments of inertia.

The nature of the deformation of a nuclear system may be deduced from its experimental spectrum and associated quadrupole moment. The relative (dimensionless) quadrupole moment $Q$ is defined as

$$
Q=\frac{1}{R_{0}^{5}} \iiint\left(3 z^{2}-r^{2}\right) d \tau
$$

(the $\mathrm{z}$ axis being the axis of revolution).

The deformation parameter $\beta$, often used in nuclear spectroscopy, follows

$$
\begin{aligned}
& \beta=\frac{0.75}{\sqrt{5 \pi}} Q R_{0}^{2}\left\langle r^{2}\right\rangle^{-1}, \\
& \beta=2 \sqrt{\frac{\pi}{5}} \frac{I_{\perp}-I_{\|}}{I_{\perp}+0.5 I_{\|}} .
\end{aligned}
$$

\section{ELLIPSOIDS}

Most of the nuclei and planets are slightly ellipsoidally deformed by rotation. In polar coordinates the axially symmetric ellipsoids are defined by

$$
1 / R(\theta)^{2}=\sin ^{2} \theta / a^{2}+\cos ^{2} \theta / c^{2}
$$

where $a$ is the transverse semi-axis and $c$ is half the elongation on the axis of revolution. The volume conservation implies $a^{2} c=R_{0}^{3}$, where $R_{0}$ is the radius of the sphere (see Fig. 1).

The prolate deformation is characterized by $s<1$ and the related eccentricity is $e^{2}=1-s^{2}$ while in the oblate case $s>1$ and $e^{2}=1-s^{-2}[18,20,21]$.

In the prolate case, the relative surface function is given by

$$
B_{s}=\frac{\left(1-e^{2}\right)^{1 / 3}}{2}\left[1+\frac{\sin ^{-1}(e)}{e\left(1-e^{2}\right)^{1 / 2}}\right]
$$

and in the oblate case

$$
B_{s}=\frac{\left(1+\epsilon^{2}\right)^{1 / 3}}{2}\left[1+\frac{\ln \left(\epsilon+\left(1+\epsilon^{2}\right)^{1 / 2}\right)}{\epsilon\left(1+\epsilon^{2}\right)^{1 / 2}}\right] \quad \epsilon^{2}=s^{2}-1 .
$$

The relative Coulomb function is, in the prolate case

$$
B_{C}=\frac{\left(1-e^{2}\right)^{1 / 3}}{2 e} \ln \left(\frac{1+e}{1-e}\right),
$$




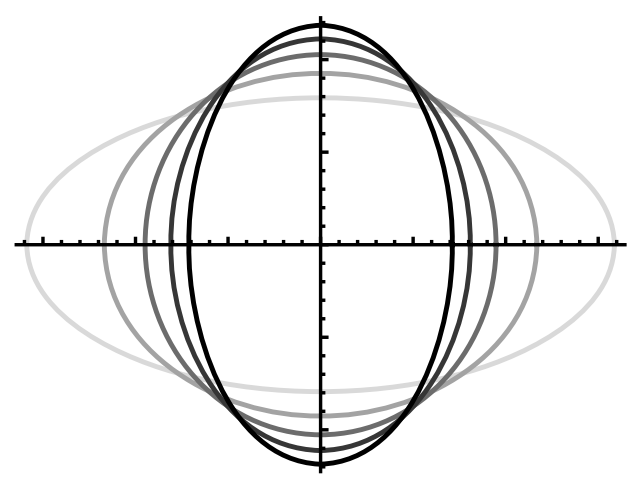

FIG. 1: Evolution of the ellipsoid configuration as a function of the ratio of the minor and major semi-axes $s=a / c$. The horizontal axis is the common axis of revolution $(\phi=0)$. $\mathrm{s}$ is respectively: $1 / 3,2 / 3,1,4 / 3$ and $5 / 3$. The ellipsoid is prolate when $s<1$ and oblate for $s>1$.

and, in the oblate case

$$
B_{C}=\frac{\left(1+\epsilon^{2}\right)^{1 / 3}}{\epsilon} \tan ^{-1} \epsilon
$$

The relative curvature function is, in the prolate case

$$
B_{k}=\frac{1}{2\left(1-e^{2}\right)^{1 / 3}}+\frac{\left(1-e^{2}\right)^{2 / 3}}{4 e} \ln \left(\frac{1+e}{1-e}\right),
$$

and, in the oblate case

$$
B_{k}=\frac{1}{2\left(1+\epsilon^{2}\right)^{1 / 3}}+\frac{\left(1+\epsilon^{2}\right)^{2 / 3}}{2 \epsilon} \tan ^{-1} \epsilon .
$$

For the prolate ellipsoidal shapes, one simply has

$$
\begin{gathered}
I_{\perp}=\frac{s^{-4 / 3}+s^{2 / 3}}{2}, \\
I_{\|}=s^{2 / 3}, \\
Q=\frac{8 \pi}{15}\left(s^{-4 / 3}-s^{2 / 3}\right) .
\end{gathered}
$$

\section{ELLIPTIC LEMNISCATOIDS}

To describe the fusion and fission processes, it is necessary to define a shape sequence evolving from one sphere to two tangent spheres or vice-versa, assuming volume conservation [17, 22]. The elliptic lemniscatoid family allows the simulation of this transition. It describes the progressive formation and suppression of a deep neck while keeping almost spherical ends. Mathematically, the elliptic lemniscatoid is the inverse of an oblate ellipsoid and the first pedal surface of a prolate ellipsoid, reciprocal of the oblate ellipsoid (see Fig. 2).

The axially symmetric prolate ellipsoid is defined as

$$
x^{2} / a^{2}+y^{2} / a^{2}+z^{2} / c^{2}=1,
$$

and its reciprocal oblate ellipsoid by

$$
a^{2} x^{2}+a^{2} y^{2}+c^{2} z^{2}=1
$$




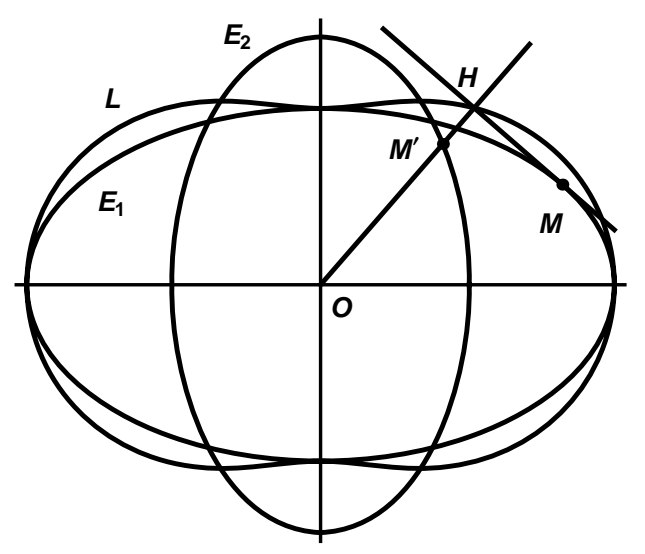

FIG. 2: Inversion leading to the elliptic lemniscatoid L. The point $\mathrm{H}$ of the elliptic lemniscatoid $\mathrm{L}$ is the projection of the origin onto the tangential plane in $\mathrm{M}$ of the prolate ellipsoid $E_{1}$. M', of the oblate ellipsoid $E_{2}$, is the inverse of the point $\mathrm{H}$. The horizontal $\mathrm{z}$ axis is the axis of revolution.

The elliptic lemniscatoid reads as

$$
a^{2} x^{2}+a^{2} y^{2}+c^{2} z^{2}=\left(x^{2}+y^{2}+z^{2}\right)^{2} .
$$

Assuming volume conservation, the unique dimensionless parameter $s=a / c$ is sufficient to completely define the shape. It is simply the ratio of the neck diameter to the system elongation. When s decreases from 1 to 0 the lemniscatoid varies continuously from a single sphere to two ones with the intermediate formation of a deep neck (see Figures 3 and 4). (These elliptic lemniscates can also generate pumpkin-like configurations when the transverse $\mathrm{x}$ axis

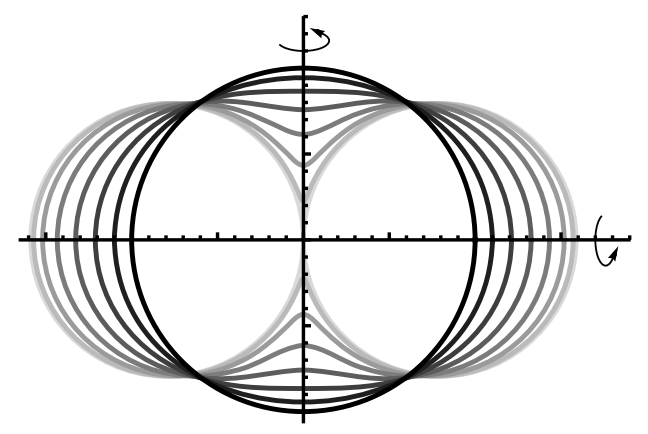

FIG. 3: Evolution of the elliptic lemniscatoid shape as a function of the ratio of the minor and major semi-axes $s=a / c$. Assuming volume conservation, the shape varies continuously from a sphere $(s=1)$ to two touching spherical fragments $(s=0)$. The neck disappears when $s>0.5 \sqrt{2}$.

is chosen as axis of revolution (see section 10)).

The axially symmetric elliptic lemniscatoid is given simply in polar coordinates (in the plane $\phi=0$ ) by

$$
R(\theta)^{2}=a^{2} \sin ^{2} \theta+c^{2} \cos ^{2} \theta
$$

The volume and surface area are given, respectively, by

$$
V=\frac{4}{3} \pi R_{0}^{3}=\frac{\pi}{12} c^{3}\left[4+6 s^{2}+\frac{3 s^{4}}{\sqrt{1-s^{2}}} \sinh ^{-1}\left(\frac{2}{s^{2}} \sqrt{1-s^{2}}\right)\right],
$$

and

$$
S=4 \pi R_{0}^{2} B_{s}=2 \pi c^{2}\left[1+\frac{s^{4}}{\sqrt{1-s^{4}}} \sinh ^{-1}\left(\frac{1}{s^{2}} \sqrt{1-s^{4}}\right)\right],
$$

where $R_{0}$ is the radius of the equivalent sphere. 


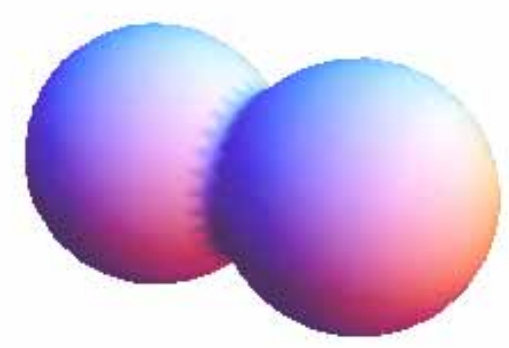

FIG. 4: 3D figure for $s=1 / 3$.

The distance $r$ between the centres of mass of the left and right parts of the system is

$$
r=\frac{2 \int_{0}^{c} z d^{3} r}{\int_{0}^{c} d^{3} r}
$$

which leads for these elliptic lemniscatoids to

$$
r=\pi c^{4} \frac{1+s^{2}+s^{4}}{3 V}
$$

The dimensionless surface curvature function is

$$
B_{k}=\frac{c}{2\left(1+s^{2}\right) R_{0}}\left[2\left(1+s^{2}\right)+\frac{s^{4}}{\sqrt{1-s^{2}}} \sinh ^{-1}\left(\sqrt{\frac{1-s^{2}}{s^{2}}}\right)-\frac{s}{\sqrt{1-s^{2}}} \tan ^{-1}\left(\sqrt{\frac{1-s^{2}}{s^{2}}}\right)\right] .
$$

The relative perpendicular and parallel moments of inertia (moment of inertia divided by $\frac{2}{5} m R_{0}{ }^{2}$, the moment of the equivalent sphere) are respectively

$$
\begin{aligned}
& I_{\perp, \text { rel }}=\frac{c^{5} s^{2}}{512\left(1-s^{2}\right) R_{0}^{5}}\left[\frac{112}{s^{2}}+8+30 s^{2}-135 s^{4}+\frac{120 s^{4}-135 s^{6}}{\sqrt{1-s^{2}}} \sinh ^{-1}\left(\sqrt{\frac{1-s^{2}}{s^{2}}}\right)\right] . \\
& I_{\|, \text {rel }}=\frac{c^{5} s^{2}}{512\left(1-s^{2}\right) R_{0}}\left[\frac{32}{s^{2}}+48+100 s^{2}-210 s^{4}+\frac{240 s^{4}-210 s^{6}}{\sqrt{1-s^{2}}} \sinh ^{-1}\left(\sqrt{\frac{1-s^{2}}{s^{2}}}\right)\right] .
\end{aligned}
$$

The relative quadrupole moment $Q$ reads as

$$
Q=\frac{\pi c^{5} s^{2}}{96\left(1-s^{2}\right) R_{0}{ }^{5}}\left[\frac{16}{s^{2}}-8-14 s^{2}+15 s^{4}-\frac{24 s^{4}-15 s^{6}}{\sqrt{1-s^{2}}} \sinh ^{-1}\left(\sqrt{\frac{1-s^{2}}{s^{2}}}\right)\right] .
$$

\section{ASYMMETRIC QUASIMOLECULAR SHAPES}

To describe the transition from two inequal spherical nuclei to one spherical nucleus or vice versa (see Fig. 5) two halves of different elliptic lemniscatoids may be joined, assuming the same transverse distance a [3, 8, 23]. Then the shape is given simply in polar coordinates (in the plane $\phi=0$ ) by

$$
R(\theta)^{2}= \begin{cases}a^{2} \sin ^{2} \theta+c_{1}^{2} \cos ^{2} \theta \quad 0 \leq \theta \leq \pi / 2 \\ a^{2} \sin ^{2} \theta+c_{2}^{2} \cos ^{2} \theta \quad \pi / 2 \leq \theta \leq \pi,\end{cases}
$$

where $c_{1}$ and $c_{2}$ are the two different half-elongations. The volume conservation is assumed, then:

$$
R_{0}{ }^{3}=R_{1}{ }^{3}+R_{2}{ }^{3},
$$




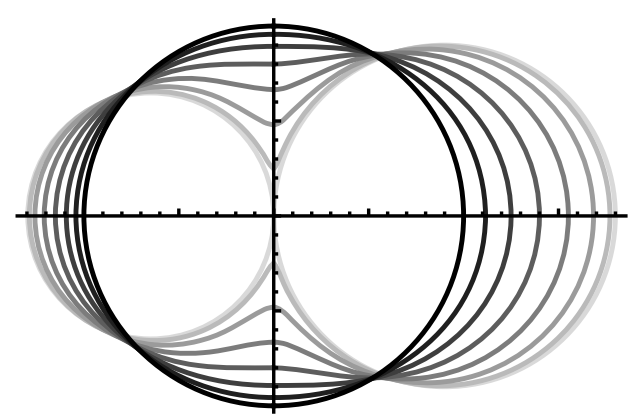

FIG. 5: Two halves of different lemniscatoids linked by a same transverse distance a, $R_{2} / R_{1}=0.72$. The horizontal axis is the axis of revolution.

where $R_{0}, R_{1}$ and $R_{2}$ are the radii of the different spheres.

The two parameters $s_{1}=a / c_{1}$ and $s_{2}=a / c_{2}$ are sufficient to completely define the shape. The ratio of the two radii $R_{2}$ and $R_{1}$ allows to connect $s_{1}$ and $s_{2}$ :

$$
s_{2}^{2}=\frac{s_{1}^{2}}{s_{1}^{2}+\left(1-s_{1}^{2}\right)\left(R_{2} / R_{1}\right)^{2}}
$$

when $s_{1}$ decreases from 1 to 0 the shape evolves continuously from one sphere to two touching different spheres with the natural formation of a deep neck, while keeping almost spherical ends.

The distance $r$ between the centres of mass of the two parts of the system is $r=r_{1}+r_{2}$. The volume of the two parts being conserved, the separation plane between them is at the distance $z_{v}$ from the origin, and $r_{1}$ and $r_{2}$ depend on $z_{v} . z_{v}$ is the solution of the equation

$$
\begin{array}{r}
\frac{1}{3} z^{3}-\frac{1}{2} a^{2} z+\frac{1}{12}\left(2 c_{2}{ }^{3}+3 a^{2} c_{2}\right)+ \\
\frac{1}{2} \sqrt{c_{2}^{2}-a^{2}}\left[D^{2} \sinh ^{-1}\left(\frac{c_{2}}{D}\right)-D^{2} \sinh ^{-1}\left(\frac{z}{D}\right)-z \sqrt{z^{2}+D^{2}}\right]=\frac{4}{3} R_{2}{ }^{3},
\end{array}
$$

with $D^{2}=a^{4} / 4\left(c_{2}{ }^{2}-a^{2}\right)$.

The positions of the mass centres $r_{1}$ and $r_{2}$ are then given by

$$
\begin{aligned}
& r_{1}=\frac{1}{\frac{4}{3} R_{1}{ }^{3}}\left\{\frac{z_{v}{ }^{4}-a^{2} z_{v}{ }^{2}}{4}+\frac{c_{1}{ }^{4}+a^{2} c_{1}{ }^{2}+a^{4}}{12}-\right. \\
& \left.\frac{a s_{2}{ }^{2}}{3\left(1-s_{2}{ }^{2}\right)}\left[\left(\frac{z_{v}{ }^{2}\left(1-s_{2}{ }^{2}\right)}{s_{2}{ }^{2}}+\frac{a^{2}}{4}\right)^{3 / 2}-\frac{a^{3}}{8}\right]\right\},
\end{aligned}
$$

and

$$
\begin{array}{r}
r_{2}=\frac{1}{\frac{4}{3} R_{2}{ }^{3}}\left\{\frac{z_{v}{ }^{4}-a^{2} z_{v}{ }^{2}}{4}-\frac{a^{4}}{4}\left(\frac{1-s_{2}{ }^{2}}{s_{2}{ }^{4}}\right)+\right. \\
\left.\frac{a s_{2}{ }^{2}}{3\left(1-s_{2}{ }^{2}\right)}\left[a^{3}\left(\frac{1}{s_{2}{ }^{2}}-\frac{1}{2}\right)^{3}-\left(\frac{z_{v}{ }^{2}\left(1-s_{2}{ }^{2}\right)}{s_{2}{ }^{2}}+\frac{a^{2}}{4}\right)^{3 / 2}\right]\right\} .
\end{array}
$$

\section{HYPERBOLIC LEMNISCATOIDS (CASSINIAN OVALOIDS)}

The scission configuration of a physical object depends strongly on its viscosity and the mean free path of its constituants. The elliptic lemniscatoids describe rather compact and creviced shapes but often the scission is a long process, occuring through elongated and little creviced shapes. This decay path can be described using hyperbolic lemniscatoids (Cassinian ovaloids) [24, 25]. In cartesian coordinates and for one-body shapes the hyperbolic lemniscatoids are defined as

$$
x^{2}=-z^{2}+0.5 c^{2}\left(s^{2}-1\right)+0.5 c \sqrt{8\left(1-s^{2}\right) z^{2}+c^{2}\left(1+s^{2}\right)^{2}} .
$$


For the two-body configurations the equation of the ovals is

$$
x^{2}=-z^{2}-0.5 c^{2}\left(s^{2}+1\right)+0.5 c \sqrt{8\left(1+s^{2}\right) z^{2}+c^{2}\left(1-s^{2}\right)^{2}} .
$$

Assuming volume conservation during the deformation, these shapes are one-parameter dependent. For the one-body configurations, the dimensionless ratio of the minor and major axes $s=a / c$ can still be chosen while, for the two-body configurations, the parameter $\mathrm{s}$ is the opposite of the ratio of the distance between the tips of the fragments and the system elongation. (see Fig. 6 and [24]). When $\mathrm{s}$ varies from 1 to -1 the shapes vary continuously from a sphere to two infinitely separated spheres. The volume of the system is, for the one-body configuration

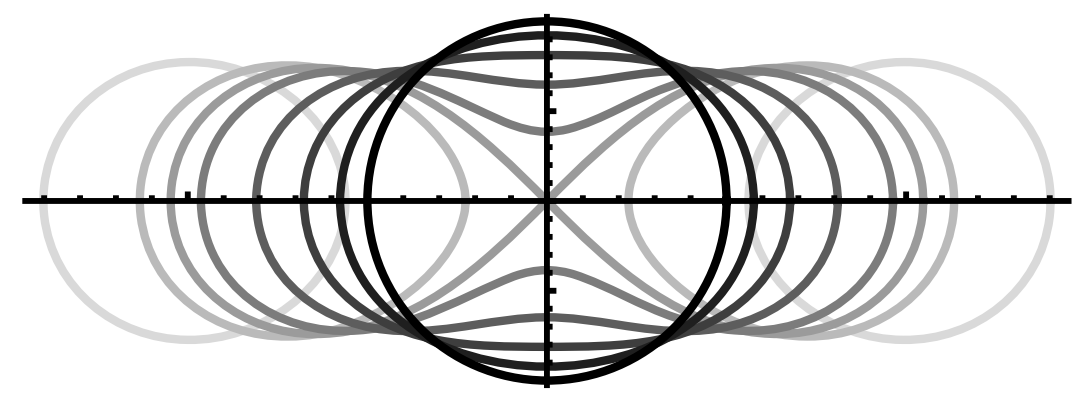

FIG. 6: Evolution of the Cassinian ovals as a function of the parameter s, assuming volume conservation. The configuration at the separation is the Bernoulli lemniscate. The shapes have a symmetry plane and a $\mathrm{z}$ axis of revolution. A neck appears when $s<\sqrt{3} / 3$.

$$
V=\frac{\pi c^{3}}{12}\left[-2+6 s^{2}+\frac{3\left(1+s^{2}\right)^{2}}{\sqrt{2\left(1-s^{2}\right)}} \sinh ^{-1}\left(\frac{2 \sqrt{2\left(1-s^{2}\right)}}{1+s^{2}}\right)\right],
$$

and for the two-body configuration

$$
V=\frac{\pi c^{3}}{12}\left[-2(1+s)^{3}+\frac{3\left(1-s^{2}\right)^{2}}{\sqrt{2\left(1+s^{2}\right)}} \sinh ^{-1}\left(\frac{2(1+s) \sqrt{2\left(1+s^{2}\right)}}{(1-s)^{2}}\right)\right] .
$$

The relative surface function for the one-body configuration is

$$
\begin{array}{r}
B_{s}=\frac{c^{2}}{4 R_{0}^{2}} \times\left[4\left(1+s^{2}\right)+2 \sqrt{\frac{2\left(1+s^{2}\right)}{1-s^{2}}} s^{2} F\left(\sin ^{-1} \sqrt{1-s^{2}}, \frac{1}{\sqrt{1+s^{2}}}\right)\right. \\
\left.-2\left(1+s^{2}\right) \sqrt{\frac{2\left(1+s^{2}\right)}{1-s^{2}}} E\left(\sin ^{-1} \sqrt{1-s^{2}}, \frac{1}{\sqrt{1+s^{2}}}\right)\right],
\end{array}
$$

where $\mathrm{E}$ and $\mathrm{F}$ are the incomplete elliptic integrals. For the two-body configuration, $B_{s}$ is calculated numerically.

The distance $r$ between the mass centre of each part is respectively for the one and two-body configurations

$$
\begin{gathered}
r=\frac{c^{4}}{8 R_{0}^{3}} \times\left(1+4 s^{2}+s^{4}\right), \\
r=\frac{c^{4}}{8 R_{0}^{3}} \times \frac{\left(1-s^{2}\right)^{3}}{1+s^{2}} .
\end{gathered}
$$

The relative parallel moment of inertia is given for the one body shape by the formula

$$
\begin{array}{r}
I_{\|}=\frac{c^{5}}{512\left(1-s^{2}\right) R_{0}{ }^{5}} \times \\
{\left[147-27 s^{2}-15 s^{4}-225 s^{6}-\frac{15\left(1+s^{2}\right)^{2}\left(15-34 s^{2}+15 s^{4}\right)}{2 \sqrt{2\left(1-s^{2}\right)}} \sinh ^{-1}\left(\frac{2 \sqrt{2\left(1-s^{2}\right)}}{1+s^{2}}\right)\right],}
\end{array}
$$


and the relative perpendicular moment of inertia by

$$
\begin{array}{r}
I_{\perp}=\frac{c^{5}}{1024\left(1-s^{2}\right) R_{0}{ }^{5}} \times \\
{\left[269+251 s^{2}-145 s^{4}-255 s^{6}-\frac{15\left(1+s^{2}\right)^{2}\left(17-30 s^{2}+17 s^{4}\right)}{2 \sqrt{2\left(1-s^{2}\right)}} \sinh ^{-1}\left(\frac{2 \sqrt{2\left(1-s^{2}\right)}}{1+s^{2}}\right)\right] .}
\end{array}
$$

The quadrupole moment is defined by

$$
\begin{array}{r}
Q=\frac{\pi c^{5}}{192\left(1-s^{2}\right) R_{0}{ }^{5}} \times \\
\left.-5+61 s^{2}-23 s^{4}+39 s^{6}+\frac{3\left(1+s^{2}\right)^{2}\left(13-38 s^{2}+13 s^{4}\right)}{2 \sqrt{2\left(1-s^{2}\right)}} \sinh ^{-1}\left(\frac{2 \sqrt{2\left(1-s^{2}\right)}}{1+s^{2}}\right)\right] .
\end{array}
$$

For the two-body configuration these shape-dependent functions are given by

$$
\begin{aligned}
& I_{\|}= \frac{c^{5}}{512\left(1+s^{2}\right) R_{0}{ }^{5}} \times\left[147+225 s+27 s^{2}-15 s^{3}-15 s^{4}+27 s^{5}+225 s^{6}+147 s^{7}\right. \\
&\left.-\frac{15\left(1-s^{2}\right)^{2}\left(15+34 s^{2}+15 s^{4}\right)}{2 \sqrt{2\left(1+s^{2}\right)}} \sinh ^{-1}\left(\frac{2(1+s) \sqrt{2\left(1+s^{2}\right)}}{(1-s)^{2}}\right)\right] 2 \sqrt{2\left(1+s^{2}\right)} \\
& I_{\perp}= \frac{c^{5}}{1024\left(1+s^{2}\right) R_{0}{ }^{5}} \times\left[269+255 s-251 s^{2}-145 s^{3}-145 s^{4}-251 s^{5}+255 s^{6}\right. \\
&\left.\left.+269 s^{7}-\frac{15\left(1-s^{2}\right)^{2}\left(17+30 s^{2}+17 s^{4}\right)}{2 \sqrt{2\left(1+s^{2}\right)}}\right)\right] \\
& Q= \frac{\pi c^{5}}{192\left(1-s^{2}\right) R_{0}{ }^{5}} \times\left[-5-39 s-61 s^{2}-23 s^{3}-23 s^{4}-61 s^{5}-39 s^{6}-5 s^{7}\right. \\
&\left.\quad+\frac{3\left(1-s^{2}\right)^{2}\left(13+38 s^{2}+13 s^{4}\right)}{2 \sqrt{2\left(1+s^{2}\right)}} \sinh ^{-1}\left(\frac{2(1+s) \sqrt{2\left(1+s^{2}\right)}}{(1-s)^{2}}\right)\right]
\end{aligned}
$$

\section{SYMMETRIC AND PROLATE TERNARY SHAPES}

From the elliptic lemniscatoids, it is possible to generate symmetric prolate ternary shapes varying from one sphere to three aligned tangent identical spheres, assuming volume conservation. Such configurations may correspond, for example, to some excited quasimolecular states of ${ }^{12} C$ formed of three aligned $\alpha$ particles [15].

While polar coordinates are very convenient in the binary case, the cartesian coordinates are more suitable to define the ternary shape sequence since the symmetry plane is no more in the necks.

The shape is defined in the first quadrant by (see Figures 7 and 8 and [26])

$$
x^{2}=0.5\left[a^{2}-2(z-d)^{2}+\sqrt{a^{4}+4(z-d)^{2}\left(c^{2}-a^{2}\right)}\right],
$$

where $\mathrm{a}$ is the neck radius and $\mathrm{c}$ is half the elongation of the generating binary case. The unique dimensionless parameter $s=a / c$ is sufficient to completely define the shape. d indicates the distance between the position of the crevice and the transverse $\mathrm{x}$ axis, namely

$$
d= \begin{cases}0.5 c \sqrt{\frac{1-2 s^{2}}{1-s^{2}}} & \text { for } 0 \leq s<0.5 \sqrt{2} \\ 0 & \text { for } 0.5 \sqrt{2} \leq s \leq 1\end{cases}
$$

The maximum transverse radial distance is

$$
h_{\text {max }}= \begin{cases}0.5 c /\left(1-s^{2}\right)^{1 / 2} & \text { for } 0 \leq s<0.5 \sqrt{2} \\ a & \text { for } 0.5 \sqrt{2} \leq s \leq 1\end{cases}
$$




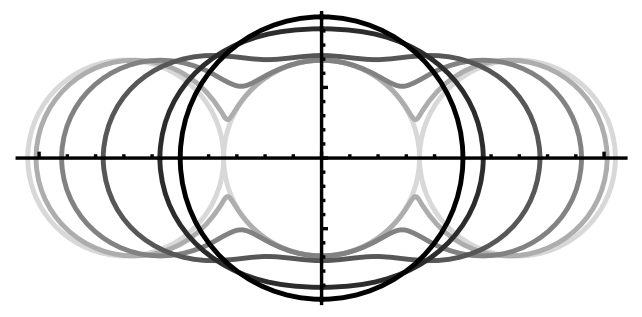

FIG. 7: Evolution of the prolate and symmetric ternary shapes as a function of the parameter s. The body has a symmetry plane and an axis of revolution. The shape varies continuously from one sphere $(s=1)$ to three aligned and touching identical spheres $(s=0)$ with the formation of two deep necks, assuming volume conservation. The neck appears when $s<0.5 \sqrt{2}$.

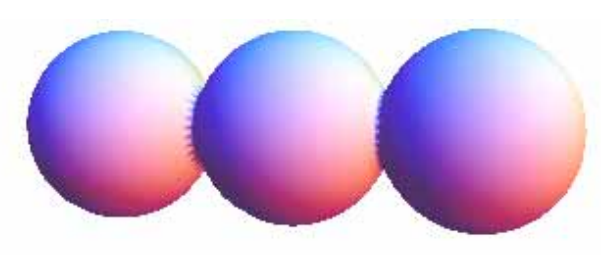

FIG. 8: 3D figure for $s=0.2$.

The volume is given by

$$
V=\frac{4}{3} \pi R_{0}^{3}=\frac{\pi c^{3}}{12}\left[4+6 s^{2}+g(\alpha)+\frac{3 s^{4}}{\sqrt{1-s^{2}}} \ln \left(\frac{2-s^{2}+2 \sqrt{1-s^{2}}}{h(\alpha)}\right)\right],
$$

where $R_{0}$ is the radius of the original sphere, $\alpha=d / c$ and

$$
g(\alpha)= \begin{cases}6 \alpha+6 \alpha s^{2}-8 \alpha^{3} & \text { for } 0 \leq s<0.5 \sqrt{2} \\ 0 & \text { for } 0.5 \sqrt{2} \leq s \leq 1\end{cases}
$$

and

$$
h(\alpha)= \begin{cases}-2 \alpha \sqrt{1-s^{2}}+1-s^{2} & \text { for } 0 \leq s \leq 0.5 \sqrt{2} \\ s^{2} & \text { for } 0.5 \sqrt{2} \leq s \leq 1\end{cases}
$$

The relative surface function is given by

$$
B_{s}= \begin{cases}\frac{c^{2}}{2 R_{0}^{2}}\left[1+\frac{\sqrt{1-2 s^{2}}}{2}+\frac{s^{4}}{\sqrt{1-s^{4}}} \ln \left(\frac{\sqrt{2}\left(1+\sqrt{1-s^{4}}\right)}{\sqrt{1-s^{2}}-\sqrt{\left(1+s^{2}\right)\left(1-2 s^{2}\right)}}\right)\right] & \text { for } 0 \leq s \leq 0.5 \sqrt{2} \\ \frac{c^{2}}{2 R_{0}^{2}}\left[1+\frac{s^{4}}{\sqrt{1-s^{4}}} \ln \left(\frac{1+\sqrt{1-s^{4}}}{s^{2}}\right)\right] & \text { for } 0.5 \sqrt{2} \leq s<1 .\end{cases}
$$

The distance between the two halves of the system is equal to

$$
r= \begin{cases}c\left(2 \alpha+\frac{\pi c^{3}\left(11-8 s^{2}\right)}{48 V\left(1-s^{2}\right)^{2}}\right) & \text { for } 0 \leq s<0.5 \sqrt{2} \\ \frac{\pi c^{4}}{3 V}\left(1+s^{2}+s^{4}\right) & \text { for } 0.5 \sqrt{2} \leq s \leq 1\end{cases}
$$

The total elongation of the system, when the three spheres are separated, is given by

$$
l=1.5 r+1.625 R_{1},
$$

where the radius of one small sphere is: $R_{1}=3^{-1 / 3} R_{0}$. The distance between the surfaces of two fragments is then given by

$$
d_{\text {frag }}=0.5 l-3 R_{1}
$$




\section{ASYMMETRIC AND PROLATE TERNARY SHAPES}

Ternary fission is much less probable than binary fission but this phenomenon has been observed, mainly via the detection of a light alpha particle and two heavier fragments. As for the elliptic lemniscatoids, the symmetric prolate ternary shapes can be generalized to describe more asymmetric shapes (see Figures 9 and 10 and [13, 19]).

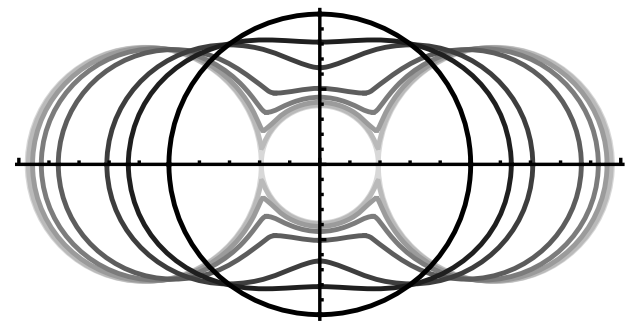

FIG. 9: Evolution of the shape from the sphere to three touching spheres. The final ratio between the radii is 0.5 .

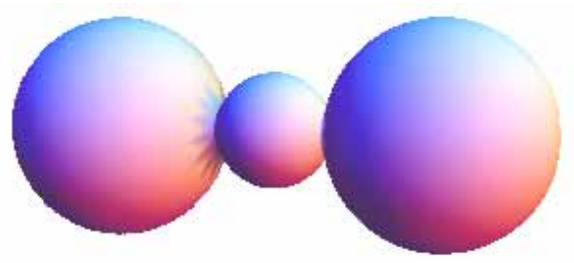

FIG. 10: 3D figure when $s_{2}=0.2$.

From these asymmetric quasimolecular shapes (see section 5), it is sufficient to introduce a symmetry plane cutting the smallest fragment along its maximal orthogonal distance and the two dimensionless parameters $s_{1}=a / c_{1}$ and $s_{2}=a / c_{2}$ are sufficient to define the shape completely. $a$ stays the neck radius and $c_{1}$ and $c_{2}$ are respectively the elongations of the external fragment and the central fragment. For $s_{1}=s_{2}=1$ the shape is only one sphere while for $s_{1}=s_{2}=0$ two spheres of radius $R_{1}$ are aligned and in contact with a central smaller sphere of radius $R_{2}$ $\left(R_{2} / R_{1} \leq 1\right)$. To connect $s_{1}$ and $s_{2}$ with the asymmetry between the central fragment and the two other external identical fragments, $s_{1}$ and $s_{2}$ may be linked by the expression

$$
s_{2}^{2}=\frac{s_{1}^{2}}{s_{1}^{2}+\left(1-s_{1}^{2}\right)\left(R_{2} / R_{1}\right)^{2}},
$$

and in the same way $c_{1}$ and $c_{2}$ are linked by

$$
c_{2}^{2}=c_{1}^{2}\left[s_{1}^{2}+\left(1-s_{1}^{2}\right)\left(R_{2} / R_{1}\right)^{2}\right]
$$

In the first quadrant the shape reads

$$
x^{2}=-(z-d)^{2}+0.5 s_{i}{ }^{2} c_{i}{ }^{2}+0.5 c_{i} \sqrt{4\left(1-s_{i}{ }^{2}\right)(z-d)^{2}+s_{i}{ }^{4} c_{i}{ }^{2}}
$$

$(i=1$ for $z>d$ and $i=2$ for $z<d)$.

The volume is now given by

$$
\begin{array}{r}
V=\frac{\pi c_{1}^{3}}{12}\left[4+6 s_{1}^{2}+\frac{3 s_{1}{ }^{2}}{\sqrt{1-s_{1}^{2}}} \sinh ^{-1}\left(\frac{2 \sqrt{1-s_{1}^{2}}}{s_{1}^{2}}\right)\right] \\
+\frac{\pi c_{2}{ }^{3}}{12}\left[6 \alpha+6 \alpha s_{2}^{2}-8 \alpha^{3}+\frac{3 s_{2}{ }^{2}}{\sqrt{1-s_{2}^{2}}} \sinh ^{-1}\left(\frac{2 \alpha \sqrt{1-s_{2}^{2}}}{s_{2}{ }^{2}}\right)\right],
\end{array}
$$

where $\alpha=d / c_{2}$. 
The surface $S=4 \pi R_{0}^{2} B_{s}$ is given by

$$
\begin{array}{r}
S=2 \pi c_{1}^{2}\left[1+\frac{s_{1}{ }^{4}}{\sqrt{1-s_{1}^{4}}} \sinh ^{-1}\left(\frac{\sqrt{1-s_{1}^{4}}}{s_{1}{ }^{2}}\right)\right] \\
+2 \pi c_{2}{ }^{2}\left[\alpha \sqrt{1-s_{2}^{2}}+\frac{s_{2}{ }^{4}}{\sqrt{1-s_{2}{ }^{4}}} \sinh ^{-1}\left(\frac{\alpha \sqrt{2\left(1-s_{2}{ }^{4}\right)}}{s_{2}{ }^{2}}\right)\right] .
\end{array}
$$

The distance between the centres of mass of the two halves of the system is

$$
r=\frac{\pi c_{1}{ }^{4}}{3 V}\left(1+s_{1}{ }^{2}+s_{1}{ }^{4}\right)+c_{2}\left[2 \alpha+\frac{\pi c_{2}{ }^{3} \alpha^{4}}{3 V} \cdot \frac{-5+8 s_{2}{ }^{2}+16 s_{2}{ }^{6}-16 s_{2}{ }^{8}}{\left(1-2 s_{2}{ }^{2}\right)^{2}}\right] .
$$

When the three aligned spherical fragments are separated :

$$
\begin{gathered}
B_{s}=\frac{2+\left(R_{2} / R_{1}\right)^{2}}{\left(2+\left(R_{2} / R_{1}\right)^{3}\right)^{2 / 3}}, \\
r=\frac{3}{2+\left(R_{2} / R_{1}\right)^{3}}\left(\frac{\left(R_{2} / R_{1}\right)^{4} R_{1}}{4}+\frac{4}{3} D\right),
\end{gathered}
$$

where $2 D$ is the distance between the centres of the two external spheres.

\section{TWO SEPARATED ELLIPSOIDS}

For separated nuclei or fragments it is often necessary to go beyond the spherical approximation and to take into account the ellipsoidal deformations (see Fig. 11).
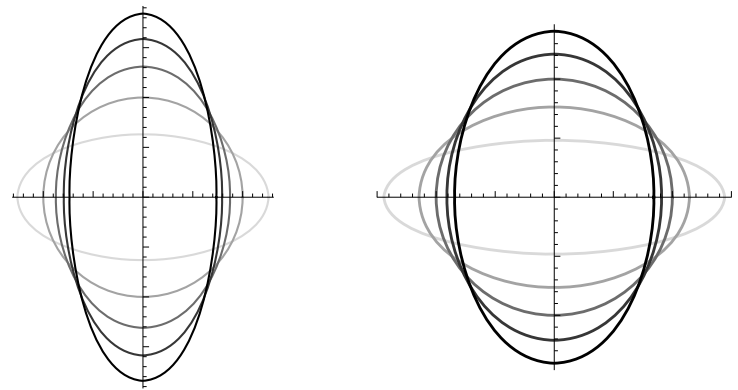

FIG. 11: Shape sequence describing the possible shape evolution of two coaxial ellipsoid configurations.

While the formulas for the volume and the surface of the binary ellipsoidal system derive directly from the formulas for one ellipsoid, the Coulomb (or gravitational) interaction energy between two coaxial ellipsoids must be calculated as $[21,27,28]$

$$
E_{C, \text { int }}(r)=\frac{Q_{1} Q_{2}}{r}\left[s\left(\lambda_{1}\right)+s\left(\lambda_{2}\right)-1+S\left(\lambda_{1}, \lambda_{2}\right)\right] \quad \lambda_{i}{ }^{2}=\frac{{c_{i}}^{2}-{a_{i}}^{2}}{r^{2}} .
$$

In the prolate case, $s\left(\lambda_{i}\right)$ is expressed as

$$
s\left(\lambda_{i}\right)=\frac{3}{4}\left(\frac{1}{\lambda_{i}}-\frac{1}{\lambda_{i}{ }^{3}}\right) \ln \left(\frac{1+\lambda_{i}}{1-\lambda_{i}}\right)+\frac{3}{2 \lambda_{i}^{2}},
$$

while, for the oblate shapes,

$$
s\left(\lambda_{i}\right)=\frac{3}{2}\left(\frac{1}{\omega_{i}}+\frac{1}{\omega_{i}^{3}}\right) \tan ^{-1} \omega_{i}-\frac{3}{2 \omega_{i}^{2}} \quad \omega_{i}^{2}=-\lambda_{i}^{2} .
$$

$S\left(\lambda_{1}, \lambda_{2}\right)$ can be calculated within a two-fold summation

$$
S\left(\lambda_{1}, \lambda_{2}\right)=\sum_{j=1}^{\infty} \sum_{k=1}^{\infty} \frac{3}{(2 j+1)(2 j+3)} \times \frac{3}{(2 k+1)(2 k+3)} \times \frac{(2 j+2 k) !}{(2 j) !(2 k) !} \lambda_{1}^{2 j} \lambda_{2}{ }^{2 k} .
$$




\section{PUMPKIN-LIKE SHAPES AND TORI}

Approximately toroidal shapes may be observed in nuclear physics, in astrophysics... Often these configurations are formed during the decay of a sphere through pumpkin-like shapes and, later, on tori and finally n-spheres emitted roughly in a plane. A pumpkin-like configuration may be simulated using elliptic lemniscates (see figure 3 ) and taking the vertical axis as axis of revolution (see Figures 12 and 13). As for the elliptic lemniscatoid, the ratio $s=a / c$ is sufficient to define the shape completely. When $s$ decreases from 1 to 0 , an hollow progressively appears in this oblate lemniscatoid, and the shape varies from a sphere to a ring torus for which the upper and lower hollows are just linked. Later on, the evolution from this initial toroidal shape towards ring torus with large radius $r_{t}$ can be governed by the dimentionless parameter $s_{t}[29,30]$

$$
s_{t}=\left(r_{t}-r_{s}\right) / 2 r_{s}
$$

where $r_{s}$ is the sausage radius and $r_{t}$ the torus radius (see Figures 13 and 14).

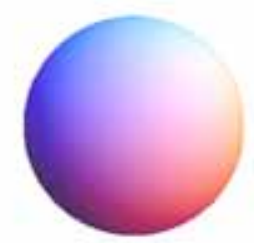

FIG. 12: Sphere or oblate elliptic lemniscatoid (pumpkin-like configuration) for $s=1$.

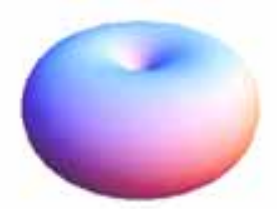

FIG. 13: Oblate elliptic lemniscatoid (pumpkin-like configuration) when $s=0$ or torus for $s_{t}=0$. The vertical axis is the axis of revolution. Volume conservation is assumed.

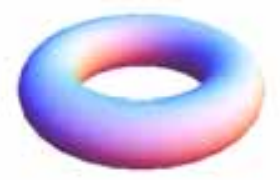

FIG. 14: Torus for $s_{t}=1$.

\section{A. Oblate elliptic lemniscatoids}

The volume, perpendicular moment of inertia, relative mean square radius and surface shape dependent function are given by

$$
V=\frac{4 \pi R_{0}^{3}}{3}=\frac{4 \pi c^{3}}{3}\left[\frac{s^{3}}{4}+\frac{3}{8}\left(s+\frac{\sin ^{-1}\left(\sqrt{1-s^{2}}\right)}{\sqrt{1-s^{2}}}\right)\right]
$$




$$
\begin{gathered}
I_{\perp, r e l}=\frac{3 c^{5}}{2 R_{0}^{5}\left(1-s^{2}\right)}\left(-\frac{s^{7}}{24}-\frac{s^{5}}{16}-\frac{25 s^{3}}{192}+\frac{35 s}{128}-\frac{5}{16}\left(s^{2}-\frac{7}{8}\right) \frac{\sin ^{-1}\left(\sqrt{1-s^{2}}\right)}{\sqrt{1-s^{2}}}\right) \\
\left\langle r^{2}\right\rangle_{r e l}=\frac{\left\langle r^{2}\right\rangle}{\frac{3}{5} R_{0}^{2}}=\frac{5 c^{5}}{4 R_{0}^{5}}\left[\frac{2 s^{5}}{15}+\frac{s^{3}}{6}+\frac{1}{4}\left(s+\frac{\sin ^{-1}\left(\sqrt{1-s^{2}}\right)}{\sqrt{1-s^{2}}}\right)\right] \\
B_{s}=\frac{s}{4 \pi R_{0}^{2}}=\frac{c^{2}}{2 R_{0}^{2}}\left(s^{2}+\frac{\sin ^{-1}\left(\sqrt{1-s^{4}}\right)}{\sqrt{1-s^{4}}}\right)
\end{gathered}
$$

\section{B. Ring torus}

For an holed torus the same quantities are defined by

$$
\begin{gathered}
V=\frac{4 \pi R_{0}^{3}}{3}=2 \pi^{2} r_{t} r_{s}^{2}=\frac{\pi^{2} c_{t}^{3}}{4}\left(1+2 s_{t}\right), \\
I_{\perp, \text { rel }}=\frac{35}{32}\left(1+3 s_{t}+3 s_{t}^{2}\right)\left(\frac{16}{3 \pi\left(1+2 s_{t}\right)}\right)^{2 / 3} \\
\left\langle r^{2}\right\rangle_{r e l}=\frac{5}{6}\left(1+2 s_{t}+2 s_{t}^{2}\right)\left(\frac{16}{3 \pi\left(1+2 s_{t}\right)}\right)^{2 / 3} \\
B_{s}=\frac{4 \pi^{2} r_{s} r_{t}}{4 \pi R_{0}^{2}}=\frac{\pi c_{t}^{2}}{4 R_{0}^{2}}\left(1+2 s_{t}\right) .
\end{gathered}
$$

It follows

$$
r_{s}=R_{0}\left(\frac{2}{3 \pi(1+2 s)}\right)^{1 / 3}
$$

\section{BUBBLES}

In heavy ion collisions at high energies, sophisticated shapes intermediate between bubbles and toroids have been formed [31]. The compression, the inhomogeneities and the out of equilibrium effects due to the violence of these collisions play an important role. Nevertheless calculations within macroscopic bubbles of constant density are railings for further investigations [30]. Assuming volume conservation, the bubble characteristics can be expressed in terms of a single parameter, the ratio $p=r_{1} / r_{2}$ of the inner radius $r_{1}$ and the outer radius $r_{2}$.

$$
\begin{gathered}
V=\frac{4 \pi R_{0}^{3}}{3}=\frac{4 \pi}{3}\left(r_{2}^{3}-r_{1}^{3}\right) \\
r_{1}=R_{0} p\left(1-p^{3}\right)^{-1 / 3} \\
r_{2}=R_{0}\left(1-p^{3}\right)^{-1 / 3}
\end{gathered}
$$

The relative (to the sphere) root mean square radius is given by

$$
\left\langle r^{2}\right\rangle_{r e l}^{1 / 2}=\frac{\left\langle r^{2}\right\rangle^{1 / 2}}{\sqrt{3 / 5} R_{0}}=\left(1-p^{5}\right)^{1 / 2}\left(1-p^{3}\right)^{-5 / 6} .
$$


The relative surface, Coulomb and moment of inertia functions read

$$
\begin{gathered}
B_{s}=\frac{1+p^{2}}{\left(1-p^{3}\right)^{2 / 3}}, \\
B_{C}=\frac{1-2.5 p^{3}+1.5 p^{5}}{\left(1-p^{3}\right)^{5 / 3}}, \\
I_{\perp, \text { rel }}=\left(1-p^{5}\right)\left(1-p^{3}\right)^{-5 / 3} .
\end{gathered}
$$

\section{SUMMARY}

Different axially symmetric shape sequences are proposed to describe ground or excited states of leptodermous nuclear matter distributions and to follow their evolution. Particularly, these configurations may simulate the path from one spherical physical object to several spherical or ellipsoidal objects in the decay and entrance channels of nuclear or astrophysical reactions (fission, fusion, fragmentation of nuclei, evolution of galaxies,...). These shapes, except the bubbles, are derived from the very rich generalized lemniscate families. Their definitions and main geometric characteristics are provided: volume, surface, root mean square radius, moment of inertia, quadrupole moment, shape dependent Coulomb funtion, ...

[1] P. Möller, J. R. Nix, W. D. Myers, and W. J. Swiatecki, At. Data Nucl. Data Tables 59, 185 (1995).

[2] K. Pomorski and J. Dudek, Phys. Rev. C 67, 044316 (2003).

[3] G. Royer and B. Remaud, Nucl.Phys. A 444, 477 (1985).

[4] G. Royer, M. Jaffré, and D. Moreau, Phys. Rev. C 86, 044326 (2012).

[5] D. N. Poenaru and W. Greiner, in Cluster in Nuclei, edited by C. Beck, Lecture Notes in Physics 818 (Springer-Verlag, Berlin Heidelberg, 2010), Vol. 1 p. 1.

[6] G. Adamian, N. Antonenko, and W. Scheid, in Cluster in Nuclei, edited by C. Beck, Lecture Notes in Physics 848 (Springer-Verlag, Berlin Heidelberg, 2012), Vol. 2 p. 165.

[7] Raj. K. Gupta, in Cluster in Nuclei, edited by C. Beck, Lecture Notes in Physics 818 (Springer-Verlag, Berlin Heidelberg, 2010), Vol. 1 p. 223.

[8] G. Royer and R. Moustabchir, Nucl.Phys.A 683, 182 (2001).

[9] D. N. Poenaru and W. Greiner, in Cluster in Nuclei, edited by C. Beck, Lecture Notes in Physics 818 (Springer-Verlag, Berlin Heidelberg, 2010), Vol. 1 p. 34.

[10] D. N. Poenaru and W. Greiner, in Cluster in Nuclei, edited by C. Beck, Lecture Notes in Physics 818 (Springer-Verlag, Berlin Heidelberg, 2010), Vol. 1 p. 45.

[11] G. Adamian, N. Antonenko, and W. Scheid, in Cluster in Nuclei, edited by C. Beck, Lecture Notes in Physics 848 (Springer-Verlag, Berlin Heidelberg, 2012), Vol. 2 p. 216.

[12] G. Royer and B. Remaud, J. Phys. G: Nucl. Part. Phys. 10, 1057 (1984).

[13] G. Royer, F. Haddad, and J. Mignen, J. Phys. G: Nucl. Part. Phys. 18, 2015 (1992).

[14] G. Adamian, N. Antonenko, and W. Scheid, in Cluster in Nuclei, edited by C. Beck, Lecture Notes in Physics 848 (Springer-Verlag, Berlin Heidelberg, 2012), Vol. 2 p. 180.

[15] G. Royer, G. Ramasamy, and P. Eudes, Phys. Rev. C 92, 054308 (2015).

[16] R. W. Hasse and W. D. Myers 1988, Geometrical Relationships of Macroscopic Nuclear Physics, (Springer verlag, Berlin, 1988).

[17] G. Royer and B. Remaud, J. Phys. G: Nucl. Part. Phys. 8, L159 (1982).

[18] G. Royer and F. Haddad, J. Phys. G: Nucl. Part. Phys. 21, 339 (1995).

[19] J. Mignen and G. Royer, J. Phys. G: Nucl. Part. Phys. 16, L227 (1990).

[20] S. Chandrasekhar, Ellipsoidal figures of equilibrium, (New Haven and London: Yale University Press, 1969).

[21] H. Schultheis and R. Schultheis, J. Math. Phys. 16, 905 (1975).

[22] B. Remaud and G. Royer, J. Phys. A: Nucl. Part. Phys. 14, 2897 (1981).

[23] G. Royer, J. Phys. G: Nucl. Part. Phys. 26, 1149 (2000).

[24] J. Mignen, G. Royer and F. Sebille, J. Phys. G: Nucl. Part. Phys. 489, 461 (1988).

[25] G. Royer and J. Mignen, J. Phys. G: Nucl. Part. Phys. 15, L1 (1989).

[26] J. Mignen and G. Royer, J. Phys. G: Nucl. Part. Phys. 13, 987 (1987).

[27] G. Royer, C. Piller, J. Mignen, and B. Remaud, Nucl.Phys. A 494, 267 (1989).

[28] G. Royer and C. Piller, J. Phys. G: Nucl. Part. Phys. 18, 1805 (1992). 
[29] C. Fauchard and G. Royer, Nucl.Phys. A 598, 125 (1996).

[30] G. Royer, F. Haddad, and B. Jouault, Nucl.Phys. A 605, 403 (1996).

[31] J. F. Lecolley et al, Phys. Lett. B 325, 317 (1994). 